\title{
Analytical insight into degradation processes of aminopolyphosphonates as potential factors that induce cyanobacterial blooms
}

\author{
Damian Drzyzga $^{1} \cdot$ Jacek Lipok ${ }^{1}$
}

Received: 10 April 2017 / Accepted: 1 September 2017 /Published online: 10 September 2017

(C) The Author(s) 2017. This article is an open access publication

\begin{abstract}
Aminopolyphosphonates (AAPs) are commonly used industrial complexones of metal ions, which upon the action of biotic and abiotic factors undergo a breakdown and release their substructures. Despite the low toxicity of AAPs towards vertebrates, products of their transformations, especially those that contain phosphorus and nitrogen, can affect algal communities. To verify whether such chemical entities are present in water ecosystems, much effort has been made in developing fast, inexpensive, and reliable methods for analyzing phosphonates. However, unfortunately, the methods described thus far require time-consuming sample pretreatment and offer relatively high values of the limit of detection (LOD). The aim of this study was to develop an analytical approach to study the environmental fate of AAPs. Four phosphonic acids, $N, N$-bis(phosphonomethyl)glycine (GBMP), aminotris(methylenephosphonic) acid (ATMP), hexamethylenediamine- $N, N, N^{\prime}, N^{\prime}$-tetrakis(methylphosphonic) acid (HDTMP), and diethylenetriamine penta(methylenephosphonic) acid (DTPMP) were selected and examined in a water matrix. In addition, the susceptibility of these compounds to biotransformations was tested in colonies of five freshwater cyanobacteria - microorganisms responsible for the so-called blooms in the water. Our efforts to track the AAP decomposition were based on derivatization of $N$-alkyl moieties with $p$-toluenesulfonyl chloride (tosylation) followed by chromatographic (HPLC-UV) separation of
\end{abstract}

Responsible editor: Vitor Manuel Oliveira Vasconcelos

Jacek Lipok

jacek.lipok@uni.opole.pl

1 Faculty of Chemistry, Opole University, Oleska 48, 45-052 Opole, Poland derivatives. This approach allowed us to determine seven products of the breakdown of popular phosphonate chelators, in nanomolar concentrations and in one step. It should be noted that the LOD of four of those products, aminemethylphosphonic acid (AMPA), $N$-phosphomethyl glycine (NPMG), $N$-(methyl)aminemethanephosphonic acid (MAMPA), and $N$-(methyl) glycine (SAR), was set below the concentration of $50 \mathrm{nM}$. Among those substances, $\mathrm{N}$-(methylamino)methanephosphonic acid (MAMPA) was identified for the first time as the product of decomposition of the examined aminopolyphosphonates.

Keywords Aminopolyphosphonates $\cdot$ Pollutant transformation $\cdot$ Analytical determination $\cdot$ Cyanobacterial biodegradation $\cdot$ Water pollution $\cdot$ HPLC

\section{Introduction}

Demand for organophosphonates has intensified in recent years because thousands of tons of these compounds are consumed by industry, agriculture, and households each year (Benbrook 2016, Coupe et al. 2012, Studnik et al. 2015). Consequently, the environmental consequence of this is the annual introduction of several thousand tons of aminopolyphosphonates (AAPs), which are released mainly into surface waters (Studnik et al. 2015). Among all phosphonates, glyphosate ( $N$-phosphonomethyl glycine) is applied in millions of kilograms per year worldwide, and its main metabolite AMPA (aminomethylphosphonic acid) appears as the most important contaminant and is accompanied by identification of toxic effects of glyphosate on some water organisms (Pesce et al. 2009, Vendrell et al. 2009). For example, $N$-phosphonomethyl glycine and AMPA have been detected in Mississippi River basin at levels above $0.1 \mu \mathrm{g} \mathrm{L} \mathrm{L}^{-1}$ 
(Coupe et al. 2012). Additionally, glyphosate accumulation in tissues of some water organisms, including Daphnia pulex, snails (Helix aspersa), carp (Cyprinus carpio), and tilapia (Oreochromis mossambicus) (Annett et al. 2014), have been reported. In 2015, glyphosate was classified as a "probable human carcinogen" by the International Agency for Research on Cancer (IARC 2015).

In comparison with glyphosate and AMPA, the knowledge on environmental fate and ecological interactions of aminopolyphosphonates that are commonly used as complexones of metal ions and as flame retardants is very scarce. More surprising is that the worldwide demand for these compounds reaches millions of tons annually (Studnik et al. 2015). Aminopolyphosphonates possess a nitrogen atom to which methylphosphonic motifs are anchored. Therefore, it is expected that such complexones may be, in a way, similar to glyphosate. Microbial biodegradation of such substances was reported with respect to mycelial fungus Aspergillus terreus (Lenartowicz et al. 2015) and cyanobacteria (Forlani et al. 2013 , 2008,2011, Lipok et al. 2010), with aminopolyphosphonates serving as the source of nutritive phosphorus for these microorganisms. Obviously, the patterns of decomposition of individual compounds vary depending on their structure (Drzyzga et al. 2017). Some of these compounds have been reported as being sensitive to chemical decomposition in water, in the presence of $\mathrm{Fe}$ (III) and Mn(II) ions, which serve as catalysts (Nowack 2003). However, their decomposition pathways have not been studied.

Independent of the degradation method, phosphorus that is present in structures of phosphonates enriches total $\mathrm{P}$ content in water reservoirs, which leads to their eutrophication (Sharpley and Wang 2014). The U.S. Environmental Protection Agency described this phenomenon as one of the main problems that impair water quality (US Environmental Protection Agency 2011). Frequently, this results in blooms of cyanobacteria and harmful algae. Cyanobacteria, also called blue-green algae, are the most widely distributed groups of photosynthetic prokaryotes on the Earth and are the pioneering organisms that are present in almost all defined ecosystems of the planet, mostly inhabiting surface waters (Schopf 2000). Cyanobacteria, as key organisms in environmental nutrition cycling (BenitezNelson 2015, Heimann and Cires 2015), may respond to the presence of phosphonates by decreasing their environmental abundance through their conversion into phosphates. The precise environmental fate of these xenobiotics, however, is unknown. Currently, phosphonates (including aminopolyphosphonates) that participate in the global phosphorus cycle can be incepted by microorganisms (Van Mooy et al. 2015). Furthermore, it is important to define the routes of transformation of aminopolyphosphonates and the fate of these compounds. However, such studies are hampered by the lack of efficient analytical methods that allow tracking the chemical changes of substrate molecules.

Many techniques and procedures have been developed and successfully used, mainly in the analysis of glyphosate and AMPA: gas chromatography (GC/MS, GC/MS/MS), highperformance liquid chromatography (HPLC), capillary electrophoresis (CE), and enzyme-linked immunosorbent assay (ELISA) (Annett et al. 2014, Poiger et al. 2017, Stalikas and Konidari 2001, Toss et al. 2017). Recently, the attempts to monitor glyphosate presence using various sensors, such as supramolecular, molecular imprinted polymers, nanofibre ones, quantum dots, or conjugated polymer-based fluorescent (CPF) chemosensors, have shown great potential in detecting this compound (Gui et al. 2017). Although the analysis of other phosphoric pesticides or phosphonic derivatives has been reported (Skeff et al. 2016, Stalikas et al. 2000), despite the use of various detection and derivatization techniques (Koskinen et al. 2016), the analysis of aminophosphonic acids in water is still extremely difficult. The main reasons are their hydrophilic character and the lack of specific chromophore in their structures (Nowack 2003). High water solubility with simultaneous poor solubility in organic solvents makes phosphonic acid derivatives extremely hard to extract from environmental matrices (Stalikas and Konidari 2001). Many efforts have been undertaken to develop fast, inexpensive, and reliable methods for their analysis (Esser et al. 2007, Nowack 1997, 2002, 2003) but without spectacular success. The LOD values of those methods were above $1 \mu \mathrm{M}$, which makes them unsuitable for application to natural samples. The application of phosphorus nuclear magnetic resonance $\left({ }^{31} \mathrm{P} N \mathrm{NM}\right)$ allowed tracing the fate of glyphosate in a culture of cyanobacterium Spirulina platensis (Lipok et al. 2007). However, the use of this technique in complex matrices is confined by high detection limits, which are additionally increased in the presence of paramagnetic metal ions in the samples.

Bearing in mind the abovementioned information, it can be stated that increasing and uncontrolled loading of aminophosphonates into the environment, combined with the ability of cyanobacteria to transform these compounds, resulted in the appearance of AAP degradation products in the environment. Unfortunately, there is a lack of a convenient method for simultaneous determination of such derivatives. Therefore, we focused on three aspects when studying the fate of aminopolyphosphonates: (i) to adapt and to improve the already existing HPLC-UV procedure for the determination of glyphosate and AMPA in water (Khrolenko and Wieczorek 2005) to make it useful to study the presence of a wider spectrum of aminophosphonates, (ii) to track stability of these compounds in water and in media upon culturing several cyanobacteria strains, and (iii) to follow the processes of aminopolyphosphonate (bio)degradation. In this respect, interactions between four aminopolyphosphonates with 
five freshwater cyanobacteria species were assessed, using a relatively simple, inexpensive, and fast procedure, which allows detection of these biodegradation products even at a nanomolar level.

\section{Experimental}

\section{Reagents}

All chemicals, if not specified, were purchased from Avantor Performance Materials Poland S.A. (Gliwice, Poland). Water that was used to prepare all solutions was purified using the Milli-Q-RO4 system (Millipore, Bedford, MA, USA). In addition to the components of cyanobacterial media, which were of technical grade, all chemicals were of analytical grade.

\section{Aminopolyphosphonates}

Aminopolyphosphonates (Table 1) were obtained from Zschimmer \& Schwarz GmbH \& Co KG (Mohsdorf, Germany), a leading European producer of phosphonates.
Potential products of decomposition of aminopolyphosphonates

Considering that the tested aminopolyphosphonates contain a common motif $\left(-\mathrm{N}-\mathrm{CH}_{2}-\mathrm{P}(\mathrm{O})(\mathrm{OH})_{2}\right)$, it may be expected that degradation processes will result in the formation of common intermediates and/or final products. In Table 2, seven of such intermediates that contain amine functions were proposed as common intermediates in the processes of (bio)degradation of compounds that are presented in Table 1 . They were selected based on the pathways of microbial degradation of glyphosate (Lipok et al. 2007, Sviridov et al. 2015).

Standard stock solutions ( $1 \mathrm{mM}$ ) of presumable products of degradation of aminopolyphosphonates were prepared in a sterile $\mathrm{Bg} 11$ medium and were stored in the dark at $4{ }^{\circ} \mathrm{C}$. Analytical solutions were prepared by diluting stock solutions with a sterile $\mathrm{Bg} 11$ medium just before the analysis.

\section{Determination of stability of aminopolyphosphonates}

The solutions of tested compounds at concentrations of $0.1 \mathrm{mM}$ were prepared in sterile (autoclaved) Milli-Q water as well as in a Bg11 (cyanobacterial) medium. To maintain sterile conditions, appropriate amounts of each

Table 1 Studied aminopolyphosphonates with a list of their practical applications based on Studnik (Studnik et al. 2015) and manufacturer's data

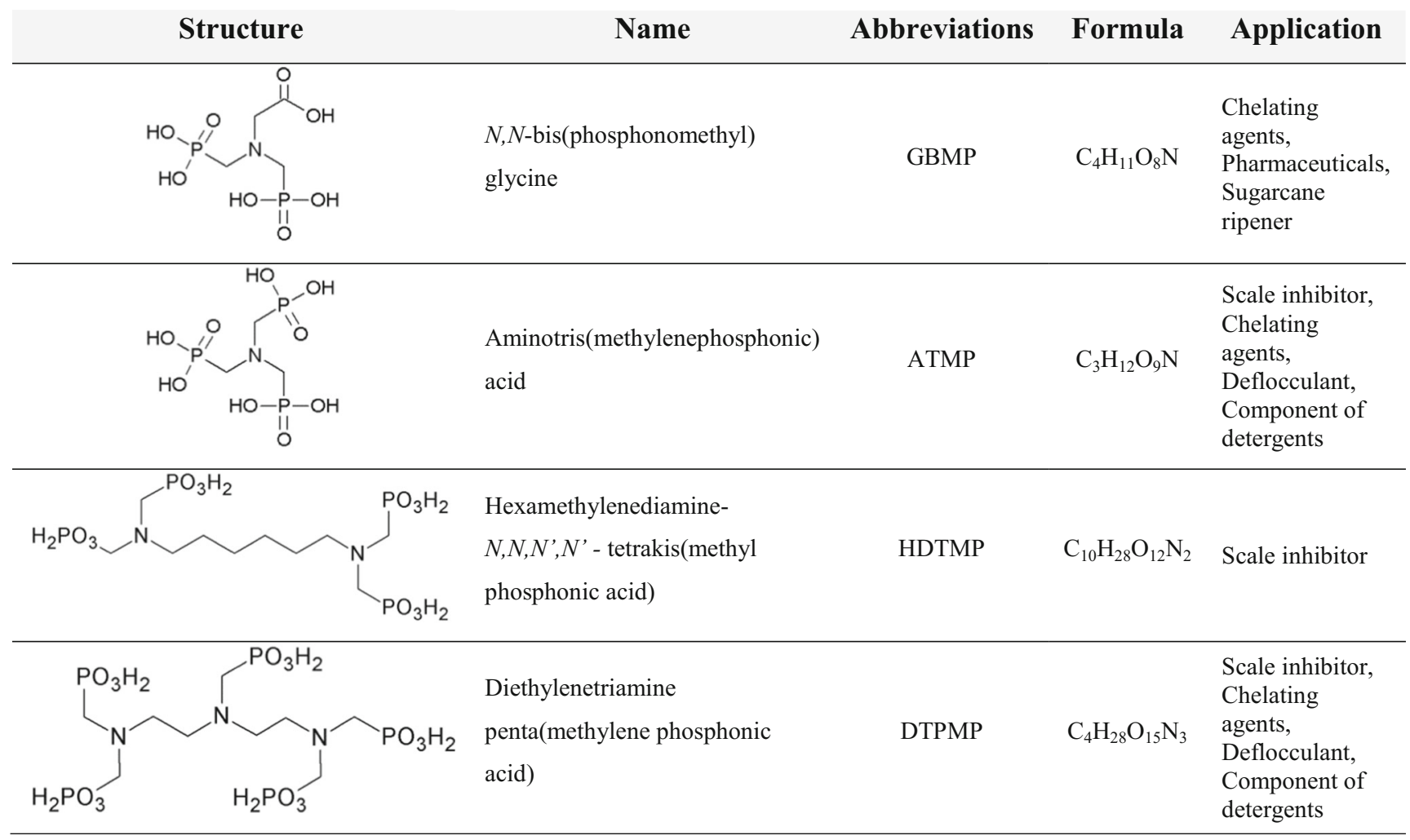


Table 2 Postulated products and intermediates of aminopolyphosphonate decomposition. Asterisk indicates this compound was a kind gift from Dr. Marta Bochno $\mathrm{PhD}$, and its synthesis is described in (Berlicki et al. 2012)

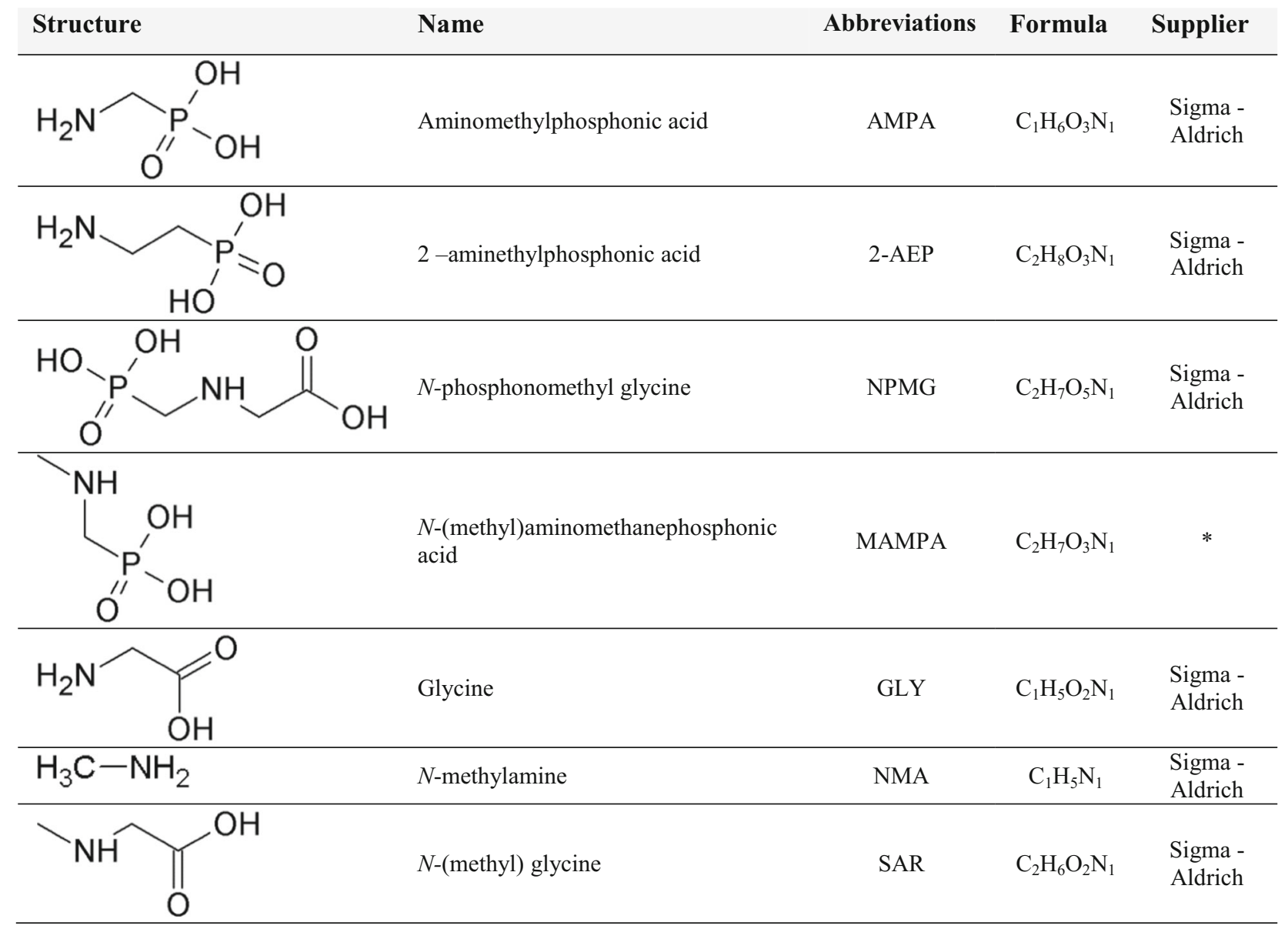

aminopolyphosphonate were dissolved in a few milliliters of sterile medium and then added to the final test solutions via filtration through a sterile membrane syringe filter (Nylon, $0.22 \mu \mathrm{m})$. Thus, the obtained sterile solutions of aminopolyphosphonates in media $(50 \mathrm{~mL})$ were placed in sterile $250-\mathrm{mL}$ Erlenmeyer flasks and maintained for at least 14 days at the same conditions as all other experiments, therefore acting as substrate controls.

\section{Sample collection and derivatization}

The $1 \mathrm{~mL}$ samples of experimental cultures of cyanobacteria, as well as the samples of substrate controls, were collected at 3-4-day intervals and were frozen in liquid nitrogen and stored at $-28^{\circ} \mathrm{C}$. On the day of analysis, appropriate samples were thawed and subjected to derivatization.

The derivatization procedure relied on tosylation of primary and secondary amine groups and was adapted from the procedure described by Khrolenko and Wieczorek (Khrolenko and Wieczorek 2005). Briefly, $150 \mu \mathrm{L}$ of the sample solution was mixed with $75 \mu \mathrm{L}$ of $0.4 \mathrm{M} \mathrm{K}_{2} \mathrm{HPO}_{4}(\mathrm{pH} 11$ ), and $30 \mu \mathrm{L}$ of $p$-toluenesulfonyl chloride $\left(10 \mathrm{mg} \mathrm{mL}^{-1}\right.$ in $\mathrm{MeCN}$ ) was added. Then, the reaction mixture was heated in water bath at $50{ }^{\circ} \mathrm{C}$ for $10 \mathrm{~min}$. Derivatization was terminated by adding $32.6 \mu \mathrm{L}$ of $1 \mathrm{M} \mathrm{HCl}$ to each sample.

\section{Chromatographic (HPLC) separation of analytes}

The samples were analyzed using a Thermo Scientific Dionex Ultimate ${ }^{\circledR} 3000$ HPLC system, equipped with a Photodiode Array Detector (PDA-3000) that monitors the eluate at $240 \mathrm{~nm}$. The derivatized samples $(20 \mu \mathrm{L})$ were injected onto a $4.6 \mathrm{~mm} \times 250 \mathrm{~mm}$ Phenomenex Kinetex $\circledR$ Evo 100-5 C-18 column connected with a dedicated SecurityGuard TM ULTRA Cartridge System. HPLC separations were performed using a mobile phase that consists of a $10 \mathrm{mM} \mathrm{KH}_{2} \mathrm{PO}_{4}$ buffer (adjusted to $\mathrm{pH} 2.3$ with $85 \% \mathrm{H}_{3} \mathrm{PO}_{4}$ ) and acetonitrile with the initial ratio of 90:10 $(v / v)$, respectively. During the first $17 \mathrm{~min}$, the composition of the mobile phase was unchanged, but the flow rate increased from $1.0 \mathrm{~mL} \mathrm{~min}^{-1}$ and reached the maximum of $1.3 \mathrm{~mL} \mathrm{~min}^{-1}$ at $17 \mathrm{~min}$. From 17 to $24 \mathrm{~min}$, the contribution of phosphate buffer was reduced to $75 \%$ 
$\left(2.14 \% \mathrm{~min}^{-1}\right)$, which was accompanied by the decrease in the flow rate to $1.0 \mathrm{~mL} \mathrm{~min}{ }^{-1}$. Next, during the time from 24 to 31 min of analysis, the composition of the mobile phase was gradually returned to initial parameters with a similar velocity, with the flow rate of $1.0 \mathrm{~mL} \mathrm{~min}{ }^{-1}$. These conditions were maintained up to the 36 th minute, when the separation was finished. Elution proceeded at $35{ }^{\circ} \mathrm{C}$. The samples of stock solutions were run with a six-fold repetition, whereas experimental samples were analyzed at least in triplicate.

\section{Validation of the analytical procedure}

The following features of the HPLC-PDA method, such as linearity, limit of detection (LOD), limit of quantitation (LOQ), and precision, were investigated and described. Validation was performed both in a fresh Bg11 medium and in a post-cultured BG11 medium. LOD and LOQ were computed based on the standard deviation of the response and slope $(\mathrm{LOD}=3.3 \sigma / s$, where $\sigma-\mathrm{S}$.D. of response, $s-$ slope of calibration curve). Limit of quantification (LOQ) was expressed as a three-fold of LOD or as LOQ $=10 \mathrm{\sigma} / \mathrm{s}$. Additionally, the impact of freezing stock solutions on LOD and LOQ values was assessed. On the day of analysis, the stock solution mixtures of $N$-containing compounds were prepared in a fresh, sterile Bg11 medium at a concentration of $1 \mathrm{mM}$. The same Bg11 medium was used to prepare a set of solutions of the tested compounds and products at a desired concentration range. All prepared solutions were frozen in liquid nitrogen and maintained at $-28{ }^{\circ} \mathrm{C}$ for 7 days. Then, they were thawed and analyzed again. Post-cultured medium was applied as a matrix. It was prepared as follows. After 14 days of culturing freshwater cyanobacterium Anabaena variabilis in a $\mathrm{Bg} 11$ medium, microbial cells were removed via centrifugation $(5000 \times \mathrm{g} / 5 \mathrm{~min})$, and the standards were dissolved in the obtained supernatant to reach the final concentration of $1 \mathrm{mM}$. Post-cultured Bg11 medium was applied to dilute the stock solutions, similar to the fresh $\mathrm{Bg} 11$ medium. The calibration curves were constructed based on six to eight points, with six injections for each concentration of each standard solution.

\section{Cyanobacterial cultures}

Cyanobacterial strains were obtained from the Culture Collection of Autotrophic Organisms at the Institute of Botany of the Academy of Sciences of the Czech Republic. The following species were examined: Anabaena variabilis (CCALA 007), Chroococcidiopsis thermalis (CCALA 049), Chroococcus minutus (CCALA 055), Fischerella cf. maior (CCALA 067), and Nostoc cf. muscorum (CCALA 129). These strains have been chosen due to their participation in cyanobacteria blooms of water bodies. Microorganisms were grown at $25 \pm 1{ }^{\circ} \mathrm{C}$ under $16 \mathrm{~h}: 8 \mathrm{~h}$ (day:night) in 250-mL
Erlenmeyer flasks containing $60 \mathrm{~mL}$ of a $\mathrm{Bg} 11$ medium (ATCC 616) (Allen 1968, Rippka 1979). To maintain cell vitality, every 21 days, the cultures were revitalized (by transferring $10-\mathrm{mL}$ aliquots to $50 \mathrm{~mL}$ of fresh medium). Experimental cultures were started in $50 \mathrm{~mL}$ of solutions of aminopolyphosphonate in a Bg11 medium, followed by the addition of cells of cyanobacteria to obtain the final concentrations of chlorophyll of $1 \mathrm{mg} \mathrm{L}^{-1}$. The chlorophyll content was determined using the already described method (Lipok et al. 2010).

To assess whether the presence of inorganic phosphate can affect the explanation of the AAP stability and the microbial ability to degrade inorganic phosphate, additional experiments were performed. A standard Bg11 medium was modified by removing $\mathrm{K}_{2} \mathrm{HPO}_{4}(\mathrm{Bg} 11-\mathrm{P})$; in this case, it was necessary to balance the potassium concentration by providing it in the form of $\mathrm{KNO}_{3}\left(34 \mathrm{mg} \mathrm{L}^{-1}\right)$, which resulted in the reduction of the amount of $\mathrm{NaNO}_{3}$ to $1.47 \mathrm{~g} \mathrm{~L}^{-1}$ (to balance the amount of $\mathrm{N}$ ).

\section{Results and discussion}

\section{Adaptation and optimization of the HPLC-based determination procedure of amino derivatives}

Because the application of HPLC chromatographic conditions, which have been already described, did not allow effective separation of all seven predicted intermediates or final products of AAP degradation; several factors, such as the flow rate, mobile phase composition, and column temperature, were optimized. The introduction of two gradient domains to isocratic separation [the first one after $17 \mathrm{~min}$, which coincides with the stable flow of mobile phase $\left(1.3 \mathrm{~mL} \mathrm{~min}^{-1}\right)$, and the second from 24 to $31 \mathrm{~min}$, accompanied by a gradual decrease of the flow (from 1.3 to $1.0 \mathrm{~mL} \mathrm{~min}^{-1}$ )] allowed the reduction of the entire time of HPLC separation to approximately $36 \mathrm{~min}$. The temperature of the column was set at $35^{\circ} \mathrm{C}$ and was stable during the entire process. As shown in Fig. 1, the optimized conditions assured the successful, simultaneous separation of all seven presumable products of degradation in one step.

\section{Validation of the analytical procedure}

\section{a) Linearity}

Calibration curves were constructed based on analytical values obtained for aminopolyphosphonates dissolved in a fresh, sterile Bg11 medium, after 1 week of freezing of the samples, and in fresh and frozen 14-days post-cultured media. That approach allowed us to investigate both the impact of freezing and storage of samples at $-28{ }^{\circ} \mathrm{C}$ and the possible 


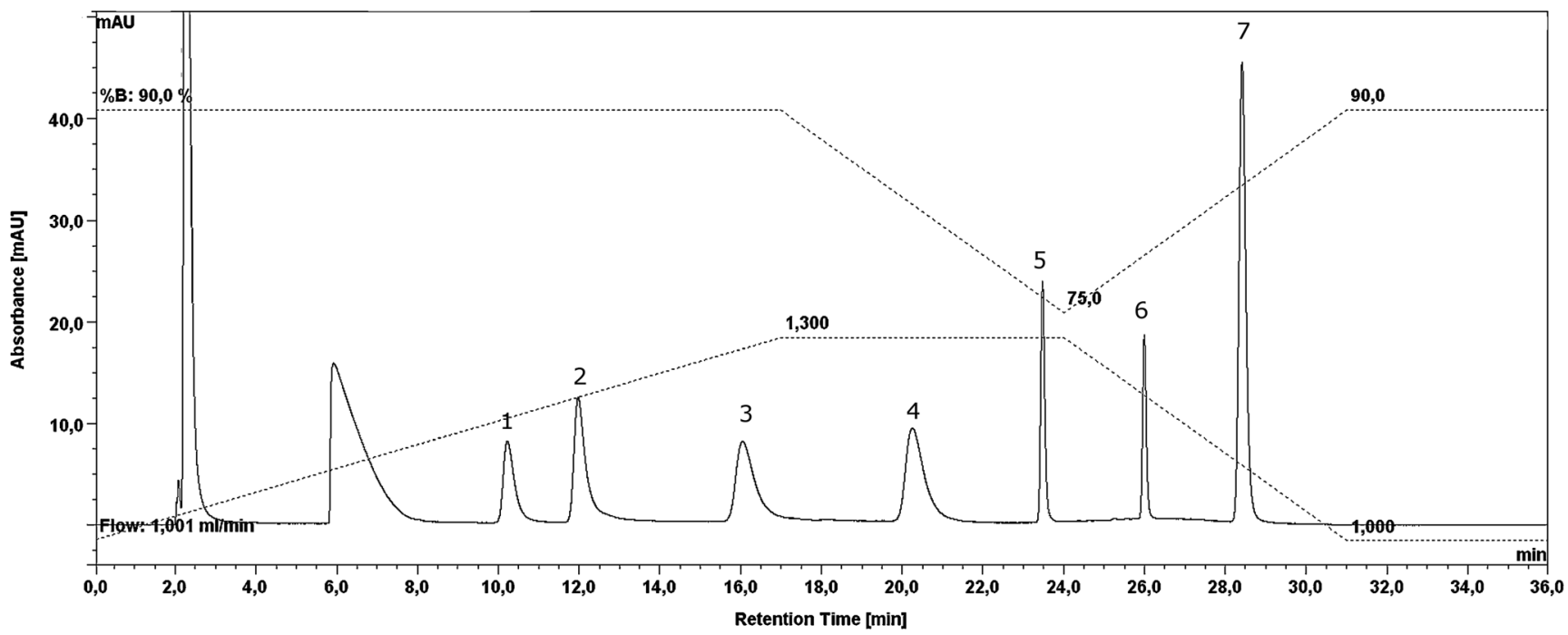

Fig. 1 Chromatogram, which presents the final separation of all seven compounds postulated as (bio)degradation products applied at a concentration of $100 \mu \mathrm{M}$ and introduced to the 14-day-old postcultured $\mathrm{Bg} 11$ medium. Amine compounds, in the form of tosyl derivatives, were separated in the following order: 1-AMPA; $2-2$ AEP; 3-NPMG; 4-MAMPA; 5-Gly; 6-NMA; 7-SAR. The

interference of analytes with microbial metabolites that are present in a post-culture media. Calibration curves for aminomethylphosphonic acid (AMPA), $N$-(methyl)aminomethanephosphonic acid (MAMPA), and $\mathrm{N}$-phosphomethyl glycine (NPMG) were found to be linear in the concentration range from 0.8 to $100 \mu \mathrm{M}$. For $2-$ aminethylphosphonic acid (2-AEP) and glycine (Gly), the curves were linear in the range of 1.5 up to $100 \mu \mathrm{M}$, while for $N$-methylamine (NMA) and $N$-(methyl) glycine (SAR), the linearity was observed from 0.4 to $100 \mu \mathrm{M}$. The values of limits of detection (LOD), limits of quantification (LOQ), and correlation coefficients are given in Table 3 .

The table includes the values of limit of detection and limit of quantification [expressed in $(\mu \mathrm{M})$ ] that are computed for stock solutions prepared in a fresh $\mathrm{Bg} 11$ medium (Bg11), after 7 days of freezing (Bg11 frozen), and stock solutions made in a 14-day-old post-cultured medium of Anabaena variabilis with cells being removed prior to the measurement (post-cultured $\mathrm{Bg} 11$ ).

The impact of freezing or the presence of cyanobacterial metabolites on the limit of detection was slight. The presence of cyanobacterial matrix had evoked the changes in the level of determination of MAMPA and SAR (compared with a Bg11 stock solution). Nevertheless, this difference amounted in only circa $10 \mathrm{nM}$. Freezing, in turn, influenced the LOD values mostly in the case of Gly (almost $50 \mathrm{nM}$ ). Nevertheless, the values of LOD and LOQ that are presented in Table 3 are of the same order of magnitude and are in a nanomolar concentration range. It should be noted that AMPA, NPMG, MAMPA, and SAR can be detected chromatogram also contains the conditions of separation marked as a dotted line. The lower one corresponds to the flow rate, while the upper one presents time-dependent participation of a $\mathrm{KH}_{2} \mathrm{PO}_{4}$ buffer in a mobile phase composition, with respect to $\mathrm{MeCN}$. The unnumbered peak with a retention time of approximately 6 min is attributed to $p$-toluenesulfonyl chloride

below the concentration of $50 \mathrm{nM}$. In regard to 2-AEP, Gly, and NMA, the detection levels were estimated in

Table 3 Computed LOD, LOQ, and $R^{2}$ values of compounds considered potential products of aminopolyphosphonate (bio)degradation. LOD and LOQ are expressed as $(\mu \mathrm{M})$

\begin{tabular}{lllll}
\hline Compound & Medium & LOD & LOQ & $R^{2}$ \\
\hline AMPA & Bg11 & 0.024 & 0.073 & 0.9999 \\
& Bg11 (frozen) & 0.026 & 0.077 & 0.9997 \\
& Post-cultured Bg11 & 0.028 & 0.083 & 0.9996 \\
& Bg11 & 0.158 & 0.474 & 0.9998 \\
& Bg11 (frozen) & 0.149 & 0.447 & 0.9999 \\
NPMG & Post-cultured Bg11 & 0.160 & 0.481 & 0.9998 \\
& Bg11 & 0.044 & 0.132 & 1.0000 \\
& Bg11 (frozen) & 0.050 & 0.151 & 0.9997 \\
MAMPA & Post-cultured Bg11 & 0.049 & 0.147 & 1.0000 \\
& Bg11 & 0.035 & 0.104 & 0.9998 \\
& Bg11 (frozen) & 0.038 & 0.114 & 0.9999 \\
Gly & Post-cultured Bg11 & 0.045 & 0.135 & 0.9994 \\
& Bg11 & 0.112 & 0.337 & 0.9999 \\
& Bg11 (frozen) & 0.159 & 0.476 & 0.9994 \\
& Post-cultured Bg11 & 0.132 & 0.395 & 0.9996 \\
NMA & Bg11 & 0.111 & 0.332 & 0.9999 \\
& Bg11 (frozen) & 0.128 & 0.385 & 0.9997 \\
& Post-cultured Bg11 & 0.119 & 0.357 & 0.9993 \\
& Bg11 & 0.041 & 0.121 & 0.9999 \\
& Bg11 (frozen) & 0.045 & 0.149 & 0.9999 \\
& Post-cultured Bg11 & 0.051 & 0.153 & 0.9995 \\
\hline
\end{tabular}


Table 4 Representative precision for $0.025-\mathrm{mM}$ stock solutions

\begin{tabular}{llll}
\hline Compound & $\begin{array}{l}\text { \%RSD Bg11 } \\
\text { medium day } \\
0\end{array}$ & $\begin{array}{l}\text { \%RSD Bg11 } \\
\text { medium } \\
\text { (frozen)-7th day }\end{array}$ & $\begin{array}{l}\text { \%RSD post-culture } \\
\text { medium Anabaena } \\
\text { variabilis }\end{array}$ \\
\hline AMPA & 3.48 & 1.45 & 2.35 \\
2-AEP & 1.44 & 1.69 & 3.79 \\
NPMG & 2.15 & 4.55 & 3.66 \\
MAMPA & 3.89 & 2.35 & 1.07 \\
Gly & 1.93 & 4.15 & 2.44 \\
NMA & 3.36 & 2.32 & 2.57 \\
SAR & 3.00 & 1.66 & 2.46 \\
\hline
\end{tabular}

Precision was expressed as the percent relative standard deviation for a statistically significant number of samples $(n=6)$

the range of $120-130 \mathrm{nM}$. Considering the simplicity of the procedure used and the complexity of the matrix, in which the compounds have been detected, the described analytical procedure seems to considerably simplify their tracking in natural waters.

\section{b) Precision of the implemented HPLC method}

The precision of the HPLC method was expressed as the percent relative standard deviation (\%RSD) and determined for $0.025-\mathrm{mM}$ stock solutions of the mixture of examined compounds (Table 4). For all batches, the values were below $5 \%$, which proved that neither the freezing process nor the presence of cyanobacterial metabolites substantially affected the precision of the HPLC-based procedure used.

\section{Stability of the tested aminopolyphosphonates in the examined liquids}

The absence of direct methods of aminopolyphosphonate determination, particularly at the concentration level below $0.1 \mu \mathrm{M}$, compels us to express their stability as a formation of new compounds. Indisputably, the components of a Bg11 medium influenced the stability of tested aminophosphonates, which resulted in the formation of some of the postulated breakdown products (Table 5.). All examined phosphonates underwent chemical decomposition that is accompanied by the release of an aminomethylphosphonic acid (AMPA) molecule but with a varied intensity. The emergence of $N$-(methyl)aminomethanephosphonic acid (MAMPA) among other decomposition products of aminotris(methylenephosphonic) acid (ATMP) and $N, N$ bis(phosphonomethyl) glycine (GBMP) should be underlined, especially because this compound has not yet been deliberated as a product of the aminopolyphosphonate transformation processes. The vulnerability of aminopolyphosphonates to degradation in Bg11 medium was characterized by the number and concentration of the formed products. Based on these data, the following order of increasing stability of tested compounds, which correlates with a growing number of methylphosphonic groups in molecules, can be concluded as follows: GBMP $<$ ATMP $<$ HDTMP $<$ DTPMP.

In addition to GBMP, which is not stable in water, instability of aminopolyphosphonates may result from the presence of some transition metal ions, as has been already stated by Nowack. It has been proven that in the presence of $\mathrm{Mn}$ (II), some AAPs undergo oxidation, whereas the presence of ions of $\mathrm{Fe}$ (II) and $\mathrm{Fe}$ (III) leads to the formation of phosphonate complexes, which undergo photodegradation (Nowack 2003). The molecule of aminotri(methylenephosphonic) acid (ATMP) subjected to oxidation in the presence of manganese ions forms iminedimethylenephosphonic acid (IDMP) and $N$-formyliminodimethylenephosphonic acid (FIDMP) (Nowack 2002).

In contrast to multifaceted and time-consuming HPLC determination, in which 9-fluorenylmethyl chloroformate (FMOC) was applied as a derivatization agent (Nowack 2002), the method applied in this work did not allow us to determine the presence of iminodi(methylenephosphonic) acid (IDMP). Although, Mn(II) ions are present in a Bg11 medium at a concentration approximately 10 times lower than the tested phosphonates, and ATMP breakdown in the presence of manganese cations was observed for equimolar amounts of these substances (Nowack and Stone 2000), the formation of iminodiphosphonic derivatives cannot be disputed. In addition, Nowack and Stone (Nowack and Stone 2000) postulated that $\mathrm{Zn}(\mathrm{II})$ and $\mathrm{Ca}$ (II) ions considerably inhibit the reaction by competing for the phosphonate substrate. In our study, these ions are present in $\mathrm{Bg} 11$ medium at a final concentration of $0.765 \mu \mathrm{M}$ and $0.24 \mathrm{mM}$, respectively, and the process of degradation still occurs. Although the $\mathrm{pH}$ of the $\mathrm{Bg} 11$ medium $(\sim 7.1)$ favors degradation via metal ion-catalyzed pathways, the same process can be hampered by other components of the medium such as EDTA and $\mathrm{Cu}$ (II) ions, which completely inhibit AAP decomposition via IDMP and FIDMP release. The appearance of an aminomethylphosphonic acid (AMPA) molecule in the solution of ATMP in a Bg11 medium (Table 5) can be related to the IDMP occurrence due to the chemical structure of the substrate. However, the composition of a Bg11 medium suppressed the appearance of AMPA with respect to GBMP. In this case, the final concentration of aminomethylenephosphonic acid was approximately ninefold lower compared with its solution in water. 
Table 5 The presence of degradation products of aminopolyphosphonates in water solutions $\left(\mathrm{H}_{2} \mathrm{O}\right)$ and in $\mathrm{Bg} 11$ medium (Bg11) after 2 weeks of storage at room temperature, expressed in micromoles per liter $(\mu \mathrm{M})$

\begin{tabular}{|c|c|c|c|c|c|c|c|}
\hline \multirow[t]{2}{*}{ Substrates } & \multicolumn{7}{|c|}{ Postulated products } \\
\hline & AMPA & 2-AEP & NPMG & MAMPA & Gly & NMA & SAR \\
\hline \multirow[t]{2}{*}{ ATMP } & $\mathrm{H}_{2} \mathrm{O}$ & - & - & - & - & - & - \\
\hline & $\operatorname{Bg} 11$ & $34.0 \pm 1.0$ & - & - & $2.9 \pm 0.4$ & - & - \\
\hline \multirow[t]{2}{*}{ GBMP } & $\mathrm{H}_{2} \mathrm{O}$ & $27.5 \pm 0.7$ & - & - & - & - & - \\
\hline & $\mathrm{Bg} 11$ & $3.0 \pm 0.3$ & - & - & $44.5 \pm 1.9$ & - & - \\
\hline \multirow[t]{2}{*}{ HDTMP } & $\mathrm{H}_{2} \mathrm{O}$ & - & - & - & - & - & - \\
\hline & Bg11 & $12.6 \pm 1.2$ & - & $-{ }^{\mathrm{a}}$ & - & - & - \\
\hline \multirow[t]{2}{*}{ DTPMP } & $\mathrm{H}_{2} \mathrm{O}$ & - & - & - & - & - & - \\
\hline & $\mathrm{Bg} 11$ & $7.8 \pm 2.2$ & - & - & - & - & - \\
\hline
\end{tabular}

-Not detected

${ }^{a}$ In the solution of HDTMP acid in a Bg11 medium, compound characterized by a retention time that is very close to NPMG, which was detected in relatively large amounts. This issue is discussed in the body of this text

\section{Tendency of aminopolyphosphonates for biodegradation}

Aminopolyphosphonates that are placed in a sole Bg11 medium have shown the tendency for spontaneous degradation, probably as a consequence of the action of transition metal ions. However, to understand the environmental fate of AAPs, it is necessary to assess their interactions with organisms that are responsible for the cycle of elements in nature. Certainly, cyanobacteria rank highly in this respect (Cottingham et al. 2015). The usefulness of several phosphonates as sources of phosphorus for aquatic microorganisms has been already proven (Drzyzga et al. 2017, Forlani et al. 2011, Ravi and Balakumar 1998, Studnik et al. 2015). Concerning the impact of cyanobacteria on the stability of AAPs, it is worth noting that among the detected intermediates, there are compounds that have been identified after 2 weeks of incubation in a sole Bg11 medium. Nevertheless, the concentrations of these substances in cyanobacterial cultures are significantly higher, even up to four- or five-fold in some cases (Table 6).

Aminotri(methylenephosphonic) acid (ATMP) molecules underwent degradation with a release of aminomethylphosphonic acid (AMPA), regardless of the presence of cyanobacteria and a deficiency in inorganic phosphate. The main metabolite of ATMP was detected in a post-culture media at a concentration of approximately $100 \mu \mathrm{M}$. Only in the culture of Chroococcidiopsis 049 was this intermediate identified at a lower concentration $(41.3 \mu \mathrm{M})$, similar to that measured in the substrate control $(34.0 \mu \mathrm{M})$. Thus, in this case, the formation of AMPA should be considered catalyzed by metal ions and not as an action of cyanobacteria. However, when examined, polyphosphonate was the sole available source of phosphorus, and aminomethylphosphonic acid was present at a concentration of approximately $127 \mu \mathrm{M}$. A similar increase in AMPA release in Bg11-P, in comparison to a Bg11 medium, was also noticed for Nostoc 129. In both media, $N$-(methyl)aminomethanephosphonic acid (MAMPA) was detected in nearly identical amounts $(\sim 3 \mu \mathrm{M})$. It is consistent with the data presented in Table 5 and indicates the lack of a microbial contribution in ATMP decomposition with the $N$-(methyl)aminomethanephosphonic acid production.

The appearance of MAMPA as the main metabolite in the biodegradation process of $N, N$-bis(phosphonomethyl) glycine (GBMP) has been proven for the first time. In a Bg11 medium, after 2 weeks, its level was approximately $44 \mu \mathrm{M}$. However, the cultivation of cyanobacteria in the presence of $100 \mu \mathrm{M}$ of GBMP led to the enhancement of the MAMPA concentration from $83 \mu \mathrm{M}$ for Chroococcus 055, even up to $156 \mu \mathrm{M}$ for Chroococcidiopsis. It can be stated that the weaker biodegradation rate of GBMP was noticed when it was the sole source of phosphorus. However, we should remember that starved microorganisms induce a number of genes that activate enzymatic pathways that are involved in $\mathrm{P}$ acquisition and assimilation (Quinn et al. 2007). Therefore, the detected lower concentration of MAMPA unambiguously does not mean a weaker decomposition of GBMP. The slightly higher AMPA and $N$-phosphomethyl glycine (NPMG) concentrations were measured after microbial treatments compared with a noninoculated $\mathrm{Bg} 11$ medium. However, this is a consequence of chemical breakdown.

It is worth emphasizing that in the case of aminotri(methylenephosphonic) acid (ATMP) and $N, N$ bis(phosphonomethyl) glycine (GBMP) subjected to both microbial and chemical degradation in a Bg11 media, cyanobacteria enhanced the formation of characteristic products of degradation several times, including AMPA for ATMP 
Table 6 Concentrations of (bio)degradation products of aminopolyphosphonates after 2 weeks of culturing with cyanobacteria

\begin{tabular}{|c|c|c|c|c|c|c|c|c|c|}
\hline \multirow[t]{2}{*}{ Substrates } & \multirow[t]{2}{*}{ Cyanobacteria strain } & \multirow[t]{2}{*}{ Medium } & \multicolumn{7}{|c|}{ Concentration of postulated products $(\mu \mathrm{M})$} \\
\hline & & & AMPA & 2-AEP & NPMG & MAMPA & Gly & NMA & SAR \\
\hline \multirow[t]{10}{*}{ ATMP } & \multirow[t]{2}{*}{ Anabaena 007} & $\mathrm{Bg} 11$ & $115.0 \pm 1.7$ & - & - & $3.2 \pm 0.5$ & - & - & - \\
\hline & & Bg11-P & $102.3 \pm 8.5$ & - & - & $2.4 \pm 0.4$ & - & - & - \\
\hline & \multirow[t]{2}{*}{ Chroococcidiopsis 049} & $\mathrm{Bg} 11$ & $41.3 \pm 0.9$ & - & - & $2.8 \pm 0.2$ & - & - & - \\
\hline & & $\mathrm{Bg} 11-\mathrm{P}$ & $126.8 \pm 2.5$ & - & - & $2.5 \pm 0.3$ & - & - & - \\
\hline & \multirow[t]{2}{*}{ Chroococcus 055} & $\mathrm{Bg} 11$ & $85.8 \pm 7.6$ & - & - & $2.9 \pm 0.4$ & - & - & - \\
\hline & & Bg11-P & $90.8 \pm 2.8$ & - & - & $3.6 \pm 0.2$ & - & - & - \\
\hline & \multirow[t]{2}{*}{ Fischerella 067} & Bg11 & $133.4 \pm 12.4$ & - & - & $3.4 \pm 0.6$ & - & - & - \\
\hline & & Bg11-P & $111.4 \pm 4.3$ & - & - & $3.9 \pm 1.5$ & - & - & - \\
\hline & \multirow[t]{2}{*}{ Nostoc 129} & $\mathrm{Bg} 11$ & $72.0 \pm 5.6$ & - & - & $3.8 \pm 1.0$ & - & - & - \\
\hline & & Bg11-P & $109.7 \pm 6.1$ & - & - & $3.3 \pm 1.4$ & - & - & - \\
\hline \multirow[t]{10}{*}{ GBMP } & \multirow[t]{2}{*}{ Anabaena 007} & Bg11 & $4.7 \pm 0.5$ & - & $1.8 \pm 0.3$ & $111.5 \pm 9.2$ & - & - & - \\
\hline & & Bg11-P & $5.4 \pm 0.3$ & - & $1.2 \pm 0.1$ & $67.3 \pm 1.8$ & - & - & - \\
\hline & \multirow[t]{2}{*}{ Chroococcidiopsis 049} & $\mathrm{Bg} 11$ & $8.3 \pm 1.1$ & - & $2.3 \pm 0.3$ & $155.8 \pm 9.8$ & - & - & - \\
\hline & & Bg11-P & $3.7 \pm 1.0$ & - & 1.00 .3 & $63.3 \pm 8.5$ & - & - & - \\
\hline & \multirow[t]{2}{*}{ Chroococcus 055} & $\mathrm{Bg} 11$ & $5.2 \pm 0.3$ & - & $1.5 \pm 0.3$ & $82.8 \pm 6.8$ & - & - & - \\
\hline & & Bg11-P & $3.0 \pm 1.0$ & - & $1.0 \pm 0.1$ & $51.5 \pm 1.2$ & - & - & - \\
\hline & \multirow[t]{2}{*}{ Fischerella 067} & $\mathrm{Bg} 11$ & $6.2 \pm 0.6$ & - & $2.3 \pm 0.2$ & $108.6 \pm 1.2$ & - & - & - \\
\hline & & Bg11-P & $4.2 \pm 0.1$ & - & $1.2 \pm 0.1$ & $65.7 \pm 0.7$ & - & - & - \\
\hline & \multirow[t]{2}{*}{ Nostoc 129} & $\mathrm{Bg} 11$ & $9.5 \pm 0.4$ & - & $2.0 \pm 0.2$ & $114.5 \pm 2.3$ & $1.5 \pm 0.1$ & $1.1 \pm 0.1$ & - \\
\hline & & Bg11-P & $5.8 \pm 0.2$ & - & $1.2 \pm 0.1$ & $73.2 \pm 3.4$ & - & - & - \\
\hline \multirow[t]{10}{*}{ HDTMP } & \multirow[t]{2}{*}{ Anabaena 007} & $\mathrm{Bg} 11$ & a & - & - & - & - & - & - \\
\hline & & Bg11-P & - & - & - & - & - & - & - \\
\hline & \multirow[t]{2}{*}{ Chroococcidiopsis 049} & $\mathrm{Bg} 11$ & $1.8 \pm 0.2$ & - & - & $0.7 \pm 0.1$ & - & - & - \\
\hline & & Bg11-P & - & - & - & - & - & - & - \\
\hline & \multirow[t]{2}{*}{ Chroococcus 055} & $\mathrm{Bg} 11$ & $2.1 \pm 0.3$ & - & - & $0.5 \pm 0.1$ & - & - & - \\
\hline & & Bg11-P & - & - & - & - & - & - & - \\
\hline & \multirow[t]{2}{*}{ Fischerella 067} & Bg11 & a & - & - & - & - & - & - \\
\hline & & Bg11-P & - & - & - & - & - & - & - \\
\hline & \multirow[t]{2}{*}{ Nostoc 129} & Bg11 & $2.5 \pm 0.1$ & - & - & - & - & - & - \\
\hline & & Bg11-P & - & - & - & - & - & - & - \\
\hline \multirow[t]{10}{*}{ DTPMP } & Anabaena 007 & Bg11 & $2.2 \pm 0.2$ & - & - & - & - & - & - \\
\hline & & Bg11-P & $1.8 \pm 0.1$ & - & - & - & - & - & - \\
\hline & Chroococcidiopsis 049 & $\mathrm{Bg} 11$ & $1.2 \pm 0.0$ & - & - & $0.5 \pm 0.0$ & - & - & - \\
\hline & & Bg11-P & $\mathrm{a}$ & - & - & - & - & - & - \\
\hline & Chroococcus 055 & Bg11 & $4.0 \pm 0.1$ & - & $\mathrm{a}$ & $1.1 \pm 0.0$ & - & - & - \\
\hline & & Bg11-P & - & - & - & - & - & - & - \\
\hline & Fischerella 067 & $\mathrm{Bg} 11$ & $14.5 \pm 1.0$ & - & - & $2.0 \pm 0.1$ & - & - & - \\
\hline & & Bg11-P & $2.3 \pm 0.2$ & - & - & $1.1 \pm 0.0$ & - & - & - \\
\hline & Nostoc 129 & $\mathrm{Bg} 11$ & $9.0 \pm 0.7$ & - & - & $1.1 \pm 0.2$ & - & - & - \\
\hline & & Bg11-P & - & - & - & - & - & - & - \\
\hline
\end{tabular}

The impact of the lack of inorganic phosphate on the accessibility of AAPs for microbes was determined (via the Bg11-P medium application). Concentrations are expressed in micromoles per liter $(\mu \mathrm{M})+$ S.D. $(n \geq 3)$. Initially, all media were fortified with the addition of aminopolyphosphonates at a final concentration of $100 \mu \mathrm{M}$. The presented values were determined in a post-cultured media after 14 days of cyanobacteria culturing using the elaborate HPLC method

-Is attributed to not detected

${ }^{a}$ The presence of an intermediate was confirmed in the experimental culture but it did not exist in a 14-day post-culture media; "0.0" - S.D. value below $0.05 \mu \mathrm{M}$ 
and MAMPA in the case of GBMP. Therefore, it can be assumed that the final effectiveness of AAP decomposition is related to the parallel action of metal ions and microbial activity. The biodegradation and chemical oxidation products are indicative of $\mathrm{C}-\mathrm{N}$ and $\mathrm{C}-\mathrm{P}$ bond cleavage.

Scale inhibitor, diethylenetriamine penta(methylene phosphonic acid) (DTPMP), during the 2 weeks of incubation in a non-inoculated $\mathrm{Bg} 11$ medium, released approximately $8 \mu \mathrm{M}$ of aminomethylphosphonic acid (AMPA). In the cultures of examined microorganisms, the amount of AMPA was lower, with an exception of Fischerella 067 in a full growth medium. Additionally, for the four out of five examined strains, small amounts of $N$-(methyl)aminomethanephosphonic (MAMPA) were detected. It is worth emphasizing that the DTPMP molecule has been already proven to be a substrate for cyanobacterial enzymes that originate from freshwater Anabaena variabilis cells (Drzyzga et al. 2017), which conceivably indicates that the entire degradation occurred intracellularly.

Interestingly, hexamethylenediamine- $N, N, N^{\prime}, N^{\prime}$ tetrakis(methyl phosphonic acid) (HDTMP) seems unlikely to be stable in cyanobacterial cultures. It has been revealed that the presence of the additional peak of retention time is very close to that of NPMG. Due to the lack of unambiguous identification of its structure, the appearance of this substance is reflected based on its amounts, which are presented in area units (mAU) (Table 7). Bearing in mind the retention time of this substance in relation to other examined compounds, it should be expected that its molecule contains a secondary amine group and a phosphonic acid motif. Therefore, the formation of (3-hydroxypropyl)aminomethylphosphonic acid, as the main product of (bio)degradation of HDTMP, may be postulated. The lower concentration of this compound, which is observed in a Bg11-P medium, should be considered the effect of Pi starvation of cells, rather than the result of a weaker decomposition rate. We postulate the appearance of a similar phenomenon for the GBMP molecule.
The stability of aminopolyphosphonates in water can be monitored by tracking the occurrence of their degradation products. The successful use of adopted and modified chromatographic procedure, which is fast, relatively inexpensive, reproducible, and requires neither expensive detector (MS, MS/MS, TOF/MS, ICP/MS, etc.), systems (e.g., UHPLC) nor time-consuming sample preparation process, has undoubtedly proven that it is possible.

\section{Conclusions}

Growing concerns about the quality of aquatic ecosystems should be associated with the implementation of methods that allow estimation of the environmental fate of aminophosphonate xenobiotics. However, due to lack of appropriate analytical procedures and because of degradation of these compounds, it is not easy to evaluate both the presence of these compounds and their influence on inhabiting biota. Degradation of aminopolyphosphonic acids may be the result of two processes: the catalytic impact of metals ions (Nowack 2002, 2003) and metabolic activity of microorganisms (Drzyzga et al. 2017). Both factors can enhance the final yield of decomposition of phosphonate. An optimized analytical procedure, which is based on derivatization (tosylation) of samples, followed by HPLC separation, has proven to be the proper tool to track this phenomenon. Furthermore, this procedure enables qualitative and quantitative determination of the set of products of degradation of aminopolyphosphonates. It is somehow surprising that, for the very first time, $\mathrm{N}$-(methyl)aminomethanephosphonic acid (MAMPA) is postulated to be the product of degradation of APPs since its chemical structure is a part of the substrate molecules. Moreover, it is possible that the compound, which was found as a new product of HDTMP (bio)degradation, can be (3hydroxypropyl)aminomethylphosphonic acid. Additionally, our results demonstrated that the metabolic activity of

Table 7 Presence of an unidentified product $(\mathrm{rt}=13 \mathrm{~min})$ expressed as an area unit $(\mathrm{mAU})$

\begin{tabular}{lllll}
\hline Substrate & Cyanobacteria strain & \multicolumn{2}{l}{ Quantity of the searched product (mAU) } \\
\cline { 3 - 5 } & & Bg11 & Post-Bg11 & Post-Bg11-P \\
\hline HDTMP & Anabaena 007 & $1.801 \pm 0.075$ & $1.429 \pm 0.411$ & $0.513 \pm 0.043$ \\
& Chroococcidiopsis 049 & & $2.300 \pm 0.115$ & $0.928 \pm 0.027$ \\
& Chroococcus 055 & & $1.885 \pm 0.134$ & $0.598 \pm 0.119$ \\
& Fischerella 067 & & $1.708 \pm 0.074$ & $0.497 \pm 0.057$ \\
Nostoc 129 & & $2.145 \pm 0.101$ & $0.332 \pm 0.144$ \\
\hline
\end{tabular}

The amounts of unidentified product were determined after 2 weeks in the media of initial addition of $0.1 \mathrm{mM} \mathrm{HDTMP}$. Quantities were expressed in area units $(\mathrm{mAU})+$ S.D. $(n \geq 3)$

$B g 11$ there was a non-inoculated medium, post-Bg11 and post-Bg11-P the HPLC analysis was performed for the post-cultured media 
freshwater cyanobacteria significantly enhanced the process of APPs degradation. As the result, the concentration of determined products has increased several times in relation to substrate controls, which was reflected by the proportional decrease in the amount of aminopolyphosphonates. However, the environmental fate of aminopolyphosphonic acids, especially the pathways of their (bio)degradation, still requires deeper investigations. The proposed relatively fast and inexpensive analytical procedure creates an opportunity for determining the set of amino derivatives in one step and at a nanomolar level. Hence, its application may enrich the knowledge about alterations in the structures of polyphosphonates that are exposed to the action of microbial communities.

Acknowledgements We thank Prof. Paweł Kafarski for his inspiration to discuss the ecological role of phosphonate compounds. The authors thank Zschimmer \& Schwarz GmbH \& Co KG (Mohsdorf, Germany) for providing aminophosphonates that are used in the study. We also thank Marta Bochno Ph D wo s ynthesized $N$ (methyl)aminomethanephosphonic acid (MAMPA).

\section{Compliance with ethical standards}

Conflict of Interest The authors declare that they have no conflict of interest.

Open Access This article is distributed under the terms of the Creative Commons Attribution 4.0 International License (http:// creativecommons.org/licenses/by/4.0/), which permits unrestricted use, distribution, and reproduction in any medium, provided you give appropriate credit to the original author(s) and the source, provide a link to the Creative Commons license, and indicate if changes were made.

\section{References}

Allen MM (1968) Simple conditions for growth of unicellular blue-green algae. J Gen Microbiol 51:199-202

Annett R, Habibi HR, Hontela A (2014) Impact of glyphosate and glyphosate-based herbicides on the freshwater environment. J Appl Toxicol 34:458-479

Benbrook CM (2016) Trends in glyphosate herbicide use in the United States and globally. Environ Sci Eur 28

Benitez-Nelson C (2015) Ocean chemistry. The missing link in oceanic phosphorus cycling? Science 348:759-760

Berlicki Ł, Bochno M, Grabowiecka A, Białas A, Kosikowska P, Kafarski P (2012) N-substituted aminomethanephosphonic and aminomethane- $P$-methylphosphinic acids as inhibitors of ureases. Amino Acids 42:1937-1945

Cottingham KL, Ewing HA, Greer ML, Carey CC, Weathers KC (2015) Cyanobacteria as biological drivers of lake nitrogen and phosphorus cycling. Ecosphere 6:art1

Coupe RH, Kalkhoff SJ, Capel PD, Gregoire C (2012) Fate and transport of glyphosate and aminomethylphosphonic acid in surface waters of agricultural basins. Pest Manag Sci 68:16-30

Drzyzga D, Forlani G, Vermander J, Kafarski P, Lipok J (2017) Biodegradation of the aminopolyphosphonate DTPMP by the cyanobacterium Anabaena variabilis proceeds via a C-P lyase-independent pathway. Environ Microbiol 19:1065-1076

Esser S, Gabelmann H, Wenclawiak BW (2007) High performance liquid chromatography for the determination of chelating agents in waste water. Anal Lett 40:1811-1819

Forlani G, Pavan M, Gramek M, Kafarski P, Lipok J (2008) Biochemical bases for a widespread tolerance of cyanobacteria to the phosphonate herbicide glyphosate. Plant Cell Physiol 49:443-456

Forlani G, Prearo V, Wieczorek D, Kafarski P, Lipok J (2011) Phosphonate degradation by Spirulina strains: cyanobacterial biofilters for the removal of anticorrosive polyphosphonates from wastewater. Enzym Microb Technol 48:299-305

Forlani G, Bertazzini M, Giberti S, Wieczorek D, Kafarski P, Lipok J (2013) Sublethal detergent concentrations increase metabolization of recalcitrant polyphosphonates by the cyanobacterium Spirulina platensis. Environ Sci Pollut Res Int 20:3263-3270

Gui M, Jiang J, Wang X, Yan Y, Li S, Xiao X, Liu T, Liu T, Feng Y (2017) Copper ion-mediated glyphosate detection with N-heterocycle based polyacetylene as a sensing platform. Sensors Actuators B Chem 243:696-703

Heimann K, Cires S (2015) N2-fixing cyanobacteria: ecology and biotechnological applications. In: Se-won K (ed) Handbook of microalgae: biotechnology advances, pp 501-515

IARC (2015) Some organophosphate insecticides and herbicides: diazinon, glyphosate, malathion, parathion, and tetrachlorvinphos. Int Agency Res Cancer Monogr 112

Khrolenko MV, Wieczorek PP (2005) Determination of glyphosate and its metabolite aminomethylphosphonic acid in fruit juices using supported-liquid membrane preconcentration method with highperformance liquid chromatography and UV detection after derivatization with p-toluenesulphonyl chloride. J Chromatogr A 1093: $111-117$

Koskinen WC, Marek LJ, Hall KE (2016) Analysis of glyphosate and aminomethylphosphonic acid in water, plant materials and soil. Pest Manag Sci 72:423-432

Lenartowicz P, Kafarski P, Lipok J (2015) The overproduction of 2,4DTBP accompanying to the lack of available form of phosphorus during the biodegradative utilization of aminophosphonates by Aspergillus terreus. Biodegradation 26:65-76

Lipok J, Owsiak T, Młynarz P, Forlani G, Kafarski P (2007) Phosphorus NMR as a tool to study mineralization of organophosphonates - the ability of Spirulina spp. to degrade glyphosate. Enzyme Microb Technol 41:286-291

Lipok J, Studnik H, Gruyaert S (2010) The toxicity of Roundup(R) 360 SL formulation and its main constituents: glyphosate and isopropylamine towards non-target water photoautotrophs. Ecotoxicol Environ Saf 73:1681-1688

Nowack B (1997) Determination of phosphonates in natural waters by ion-pair high performance liquid chromatography. J Chromatogr A 773:139-146

Nowack B (2002) Determination of phosphonic acid breakdown products by high-performance liquid chromatography after derivatization. J Chromatogr A 942:185-190

Nowack B (2003) Environmental chemistry of phosphonates. Water Res 37:2533-2546

Nowack B, Stone AT ( 2000$)$ Degradation of nitrilotris(methylenephosphonic acid) and related (amino)phosphonate chelating agents in the presence of manganese and molecular oxygen. Environ Sci Technol 34:4759-4765

Pesce S, Batisson I, Bardot C, Fajon C, Portelli C, Montuelle B, Bohatier $\mathrm{J}$ (2009) Response of spring and summer riverine microbial communities following glyphosate exposure. Ecotoxicol Environ Saf 72:1905-1912

Poiger T, Buerge IJ, Bachli A, Muller MD, Balmer ME (2017) Occurrence of the herbicide glyphosate and its metabolite AMPA 
in surface waters in Switzerland determined with on-line solid phase extraction LC-MS/MS. Environ Sci Pollut Res Int 24:1588-1596

Quinn JP, Kulakova AN, Cooley NA, McGrath JW (2007) New ways to break an old bond: the bacterial carbon-phosphorus hydrolases and their role in biogeochemical phosphorus cycling. Environ Microbiol 9:2392-2400

Ravi V, Balakumar H (1998) Biodegradation of the C-P bond in glyphosate by the cyanobacterium Anabaena variabilis L. J Sci Ind Res 57:790-794

Rippka R, Deruelles J, Waterbury JB, Herdman M, Stainer RY (1979) Generic assignment, strain histories and properties of pure cultures of cyanobacteria. J Gen Microbiol 111:1-61

Schopf JW (2000) The fossil record: tracing the roots of the cyanobacterial lineage. In: Whitton BA, Potts M (eds) The ecology of cyanobacteria. Their Diversity in Time and Space. Springer Netherlands, Amsterdam, pp 15-33

Sharpley A, Wang X (2014) Managing agricultural phosphorus for water quality: lessons from the USA and China. J Environ Sci (China) 26: 1770-1782

Skeff W, Recknagel C, Schulz-Bull DE (2016) The influence of salt matrices on the reversed-phase liquid chromatography behavior and electrospray ionization tandem mass spectrometry detection of glyphosate, glufosinate, aminomethylphosphonic acid and 2aminoethylphosphonic acid in water. J Chromatogr A 1475:64-73

Stalikas CD, Konidari CN (2001) Analytical methods to determine phosphonic and amino acid group-containing pesticides. J Chromatogr A 907:1-19
Stalikas CD, Pilidis GA, Karayannis MI (2000) An integrated gas chromatographic method towards the simultaneous determination of phosphoric and amino acid group containing pesticides. Chromatographia 51:741-746

Studnik H, Liebsch S, Forlani G, Wieczorek D, Kafarski P, Lipok J (2015) Amino polyphosphonates - chemical features and practical uses, environmental durability and biodegradation. New Biotechnol $32: 1-6$

Sviridov AV, Shushkova TV, Ermakova IT, Ivanova EV, Epiktetov DO, Leontievsky AA (2015) Microbial degradation of glyphosate herbicides (review). Appl Biochem Micro 51:188-195

Toss V, Leito I, Yurchenko S, Freiberg R, Kruve A (2017) Determination of glyphosate in surface water with high organic matter content. Environ Sci Pollut Res Int 24:7880-7888

US Environmental Protection Agency (2011) Working in partnership with states to address phosphorus and nitrogen pollution through use of a framework for state nutrient reductions

Van Mooy BA, Krupke A, Dyhrman ST, Fredricks HF, Frischkorn KR, Ossolinski JE, Repeta DJ, Rouco M, Seewald JD, Sylva SP (2015) Phosphorus cycling. Major role of planktonic phosphate reduction in the marine phosphorus redox cycle. Science 348:783-785

Vendrell E, Ferraz DG, Sabater C, Carrasco JM (2009) Effect of glyphosate on growth of four freshwater species of phytoplankton: a microplate bioassay. Bull Environ Contam Toxicol 82:538-542 